\title{
Anti-Inflammatory Effect of Xanthohumol in Combination With Praziquantel is Associated With Epithelial Mesenchymal Transition Factor Twist in Opisthorchiasis-Associated Cholangiocarcinoma
}

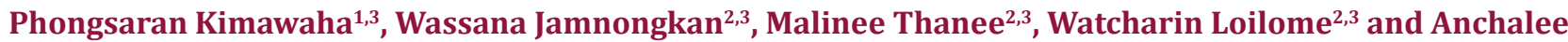 \\ Techasen $^{1,3 *}$
}

${ }^{1}$ Faculty of Associated Medical Sciences, Khon Kaen University, Thailand

${ }^{2}$ Department of Biochemistry, Faculty of Medicine, Khon Kaen University, Thailand

${ }^{3}$ Cholangiocarcinoma Research Institute, Khon Kaen University, Thailand

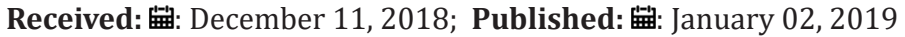

*Corresponding author: Anchalee Techasen, Department of Medical Microbiology, Faculty of Associated Medical Sciences, Khon Kaen University, Khon Kaen, 40002, Thailand
Abstract groups of hamsters were conducted including;
a) OV and NDMA treated (ON),
b) ON with praziquantel receiving (ONP),
c) ON with xanthohumol receiving (XON) and
d) ON combination with praziquantel and xanthohumol receiving (XONP) at 60, 90,120 and 180 days.

Cholangiocarcinoma (CCA) is the cancer of bile duct which is the common cancer and the major public health problem of northeastern region, Thailand. The major cause of CCA is chronic inflammation induced by liver fluke Opisthorchis viverrini (Ov) infection. Furthermore, one of key mechanism in the development of CCA is epithelial mesenchymal transition (EMT), which is characterized by loss of epithelial markers and gain of mesenchymal markers. One of the key EMT protein is Twist, an EMT transcription regulator, provides a significant prognostic marker that could be used as an EMT molecular target. Nowadays, there is no effective CCA treatment available. Several studies have attempted to use effective natural products for prevention and treatment of cancer including CCA. In this study aims to evaluate whether xanthohumol (XN) in combination with praziquantel, anthelmintic drug effect on inflammatory and Twist expression in cholangiocarcinogenesis in hamster model. Herein, four study

The results revealed that inflammatory cells around bile ducts epithelial were decreased in ONP and XONP treated when compared with ONtreated group. However, decreasing of inflammatory cells was not seen in XON-treated hamsters. In addition, higher Twist expression was observed in ON group and the strongest staining of Twist was found at day 180. Interestingly, the reduction of Twist was seen in XON and ONP groups at day 90 when compared with the ON group. Moreover, our results exhibited an effective reduction of Twist expression around bile duct area for the XONP group. In summary, XN alone could not reduce inflammatory lesions around bile duct epithelial cells but in combination with PZQ can effectively decrease of inflammatory cell in liver. For EMT, XN and/or PZQ could potentially reduce Twist expression. Further studies are needed to confirm in depth the association between Twist and CCA prevention.

Keywords: Cholangiocarcinoma; Epithelial Mesenchymal Transition; Xanthohumol; Praziquantel; Twist

Abbreviations: CCA: Cholangiocarcinoma; OV: Opisthorchis Viverrini; EMT: Epithelial Mesenchymal Transition; BHLH: Human Basic Loop Helix; PZQ: Praziquantel; NDMA: N-Nitrosodimethylamine; DAB: Diaminobenzidine Tetrahydrocholoride; IASL: International Association for the Study of the Liver; H\&E: Hematoxylin and Eosin

\section{Introduction}

Cholangiocarcinoma (CCA) is the most common liver cancer that has frequently incidence especially in the Northeast of
Thailand. This malignant is due to the infection of liver fluke Opisthorchis viverrini (Ov), which causes cancer progression 
through inflammatory process around the bile duct epithelium [1]. There is a large number of free radicals resulted in oxidative stress that has the potential to destroy the biomolecules for instance DNA, lipids and proteins, as well as the genetic alteration [2]. Furthermore, it is also found that liver fluke antigens can be diffused and attached into liver cells, bile duct epithelium and found along with the inflammatory cells occurred at the area of parasite infection [3]. Liver fluke infection can cause the pathophysiology in the liver and bile ducts with various mechanisms, a sucker of living parasite can generate the abrasion in bile duct epithelium, the secretory from the parasite which is considered for antigen be able to trigger the immune response and the inflammatory process by stimulating the immune system. Induction of inflammatory cells, such as macrophage, eosinophils and neutrophils, is also commonly found to move in areas where there is Ov infection. At the same time, these cells are stimulated to produce large amounts of free radicals.

These free radicals not only destroy parasites but they can also alter molecular biology leading to CCA genesis [4]. Another factor that contributes to CCA development is the movement of the parasite in the bile duct that can cause irritation within the bile duct cells resulting in shredded of bile duct epithelium. In combination with cell damage, free radicals cause cell death and disruption of bile duct epithelium at all times emerging the enlargement and reproduction of its cells called hyperplasia [5]. Moreover, one of key mechanism in the development of CCA is epithelial mesenchymal transition (EMT), is the process which is characterized by loss of epithelial markers and gain of mesenchymal markers that supporting cancer cell metastasis [6]. The characteristics of EMT are composed of induction of transcription factors including Twist, Snail, Slug, ZEB1 and ZEB2. The human basic-loop-helix (bHLH) Twist gene is also induces metastasis in tumor cells through the EMT process particularly in the metastasis process that Twist can facilitate intravasation [7] and invasion in various cancers for instance cervical, prostate, endometrial, [8-10] and hepatocellular carcinoma [11]. In addition, Twist overexpression is associated with the up-regulation of mesenchymal proteins in human gastric, prostate, and hepatic cancers [12].

From our previous study showed that the high expression of Twist was significantly associated with poor prognosis of CCA patients [13]. Furthermore, the correlation between Twist and inflammation has been studied and revealed that Twist proteins is the anti-inflammatory factors and pathways in regulation of TNF- $\alpha$ and provide a mechanism by which type I IFNs and Axl receptors suppress inflammatory cytokine production [14]. Thus, Twist provides a significant prognostic marker that could be used as an EMT molecular target and also as a treatment efficiency marker in CCA. At present, active drug treatment for liver fluke infection is praziquantel (PZQ), a broad-spectrum anthelmintic drug, causing parasite paralysis by increasing cell membrane permeability to calcium, resulting in contraction of the musculature of the parasite and eventually paralysis and death. Moreover, PZQ also causes the parasite to become accessible to eradicate by the host's immune response. In the treatment of CCA, the gold standard is surgical resection but is often impossible to completely resect the tumor, so there are no currently practical treatments for CCA patients.

Accumulating many studies suggests that bioactive substances from plants (phytochemicals) may be ensuring chemopreventive and chemotherapeutic agents in the treatment of several human cancers. Nowadays, medicinal plants are gaining interest in bringing to study for the treatment of various diseases, including cancer because it has a broad spectrum of action and has the potential to prevent and treat cancer effectively. Therefore, efforts are being made to find substances from natural products used in the prevention or treatment of cancer. Xanthohumol (XN; 3 ' (3,3-dimethylallyl)-2',4',4-trihydroxy-6'-methoxychalcone), the most abundant prenylated flavonoid $(0.1-1 \%$ of dry weight) in Hops (Humulus lupulus L.) which used to produce beer, a major dietary source of prenylated flavonoids, where it has been found at concentrations of up to $0.96 \mathrm{mg} / \mathrm{l}(1.95 \mu \mathrm{M})$ [15]. This substance inhibited the growth of cancer cells and increases the enzyme activity. Recently, an increasing number of studies demonstrated the broad-spectrum anticancer and prevention activity of XN in many types of cancer such as hepatocellular carcinoma, epithelial ovarian cancer [16-17], colon cancer prostate cancer [18,19].

Additionally, in vivo and in vitro studies revealed that XN can inhibit the proliferation and induced apoptosis of cancer cells. XN can suppress Ki-67 expression, CD31-positive microvessel density, NF- $\kappa$ B p65 expression, and VEGF and IL-8 levels. Taken together, these results showed that XN inhibited angiogenesis by suppressing NF- $\kappa B$ activity in pancreatic cancer [20]. Recently study reported that XN dramatically suppressed the growth of tumor and induced apoptosis in CCA cells by inhibited STAT3 activation due to suppression of the Akt-NFkB signaling pathway [21]. Moreover, our previous studies have shown that $\mathrm{XN}$ has the potential to reduce periductal fibrosis in early stages of liver fluke infection as well as efficiently reducing fibrosis in long time treatment and found the reduction of fibrosis due to Ov infection was competently eliminated in combination with PZQ treatment [22]. Thus, it is considered that $\mathrm{XN}$ is one chemopreventive substance that is effective in the prevention of cancer by inhibiting all process of the carcinogenesis [23]. These data suggested that XN has a potential to become a useful new medical approach for the chemoprevention of CCA. Finally, our study aimed to investigate the effect of XN with or without PZQ on inflammation and EMT in the expression of Twist protein during CCA genesis in a hamster model.

\section{Materials and Methods}

\section{The Preparation of Parasite for Hamster Infection}

Extraction of Ov metacercariae were achieved from naturally infected cyprinid fish which were acquired from the local market in Khon Kaen Province, northeast Thailand. The fish were chopped up and digested with pepsin-HCL, then incubated in shaking water bath at $37^{\circ} \mathrm{C}$ for an hour. After that, the digested fish were filtered through the sieves $(1,000,425$ and $106 \mu \mathrm{M}$ respectively) and it was sedimented with $0.85 \%$ saline in a sedimentation jar. Subsequently, the metacercariae were isolated and determined under a stereo 
microscope. The five male Syrian golden hamsters were fed by the total number of fifty viable active Ov cysts to each by intragastric intubation.

\section{The Preparation and Administration of N-Nitrosodime- thylamine}

The carcinogen, N-nitrosodimethylamine (NDMA) (SigmaAldrich, St. Louis MO, USA), was diluted at the concentration 12.5 ppm in distilled water. Subsequently, it was administrated daily to the assigned experiment hamster groups starting on day 30 until day 60 after the infection of $0 v$.

\section{The Preparation of Xanthohumol}

The XN was obtained from Hopsteiner, Mainberge, Germany. The preparation of XN-supplemented water was performed everyday as $1 \mu \mathrm{l}$ of stock $20 \mathrm{mM}$ in $250 \mu \mathrm{l}$ of distilled water, yielding a final concentration of $20 \mu \mathrm{M}$ or $171 \mathrm{mg} / \mathrm{B} . \mathrm{W}$./day in the experimented groups. The pre-treated with XN to hamsters were achieved more than 14 days before the experiment launched and the XN treatment was proceeded until animals were sacrificed at day 60, 90, 120 and 180 post-treatment.

\section{The Preparation of Praziquantel}

The anthelmintic drug, PZQ (Sigma-Aldrich, St. Louis MO, USA), was diluted with a non-ionic solubilizer and emulsifier that was chemophor at $2 \%$ concentration. The oral administration of PZQ was performed by a single dose of $400 \mathrm{mg} / \mathrm{kg}$ to the assigned treatment groups after day 30 of Ov infection.

\section{Experimented Animal Groups}

The study protocol was approved by the Animal Ethics Committee of Khon Kaen University (AEKKU 23/2555). The four groups of male Syrian golden hamsters (age 6-8 weeks, 2 hamsters per group) were randomly separated into as followings: group I, Ov infection and NDMA administration (ON); group II, Ov infection and NDMA administration and PZQ treatment (ONP); groups III and IV were related to group I and II by additionally received $20 \mu \mathrm{M}$ of XN (171 mg/B.W./day) designated as XON and XONP groups, respectively. Hamsters were infected with the amount of 50 Ov metacercaria by oral inoculation which was administered with NDMA at concentration $12.5 \mathrm{ppm}$ in water for 30 days and withdrawn thereafter. The preparation of XN-supplemented water was performed daily as $1 \mu \mathrm{l}$ of stock $20 \mathrm{mM}$ in $250 \mu \mathrm{l}$ of distilled water. The hamsters were killed at 60, 90, 120 and 180 days after treatment and their liver tissues were accumulated for further studies.

\section{Hematoxylin and Eosin Staining of Hamster Liver Tissue}

Hematoxylin and eosin (H\&E) staining was used for evaluation of inflammatory cells. Firstly, hamster liver sections were deparaffinized and rehydrated with graded ethanol solution. The sections were stained with hematoxylin for $6 \mathrm{~min}$, washed in distilled water for $5 \mathrm{~min}$, and rapidly washed in $70 \%$ acid ethanol, PH 2.5 at three times. After that washed in running tap water for $15 \mathrm{~min}$ and stained the sections with working eosin-phloxine solution for $2 \mathrm{~min}$. The sections were dehydrated with stepwise increasing concentration of ethanol and xylene and mounted with mounting medium. The stained sections were examined under a light microscope. The staining of H\&E can separate the components of cells by color in which hematoxylin will give blue color for the nucleus and eosin will give the pink color for the cytoplasm. The histopathological evaluation of staining was adapted using the International Association for the Study of the Liver (IASL) for inflammatory cells; Grade 1: few, Grade 2: moderate, Grade 3: numerous [24].

\section{Immunohistochemistry Staining of Twist}

Immunohistochemistry was performed to determine the expressions of Twist in hamster liver tissue. Briefly, the sections were passed through graded ethanol solutions to deparaffinize and rehydrate the sections. Antigen retrieval was performed by heating the sections in a microwave oven in $10 \mathrm{mM}$ citrate buffer $\mathrm{PH} 6.0$ for 10 minutes, while $0.3 \%(\mathrm{v} / \mathrm{v})$ hydrogen peroxide was used to block endogenous peroxidase activity. Then, 10\% skim milk in PBS was added to block non-specific substances in tissues. Each section was incubated with primary antibody; rabbit anti-Twist (Abcam, Cambridge, UK) at $4^{\circ} \mathrm{C}$ overnight. After that, sections were washed in 0.1\% Tween20 in PBS and incubated with horseradish peroxidaseconjugated EnvisionTM secondary antibody (Dako, USA). The color was developed with 3, 3' diaminobenzidine tetrahydrocholoride (DAB) substrate kit (Vector Laboratories, Ca), then counterstained with Mayer's haematoxylin. The sections were dehydrated with stepwise increasing concentration of ethanol and mounted with permount solution. The stained sections were examined under a light microscope.

\section{Statistical Analysis}

Statistical analysis was performed using SPSS software V.23.0 and Graphpad Prism V.6 to generate graphical figures. The difference in grading of inflammatory cells results was performed by student's t-test in two-tailed test. Values of $\mathrm{P}<0.05$ were considered statistically significant.

\section{Results}

The anti-inflammatory effect of XN with or without PZQ supplements on the liver histopathology of $\mathrm{Ov}$ infection and NDMA administration in hamster model

The H\&E staining was focused on the localization and aggregation of inflammatory cells surrounding the hepatic bile ducts. In the $\mathrm{ON}$ group showed a high aggregation of inflammatory cells on day 60 and decreased on day 90 and returned to increase until day 180 especially in the area where had Ov infection that was the result like the XON group because XN could not eradicate the parasite from bile ducts as shown in Figure 1. Interestingly, in XON group exhibited the numerous of inflammatory cells around bile ducts from day 60 until day 180. However, in ONP and XONP group demonstrated no or only a few inflammatory cells when compared with $\mathrm{ON}$ and XON group. From Figure 2 revealed a trend that the inflammatory cells were abundant in $\mathrm{ON}$ and XON group when compared with the treated groups. These results indicated that XN in combination with PZQ could inhibit the ongoing inflammatory process precisely. 


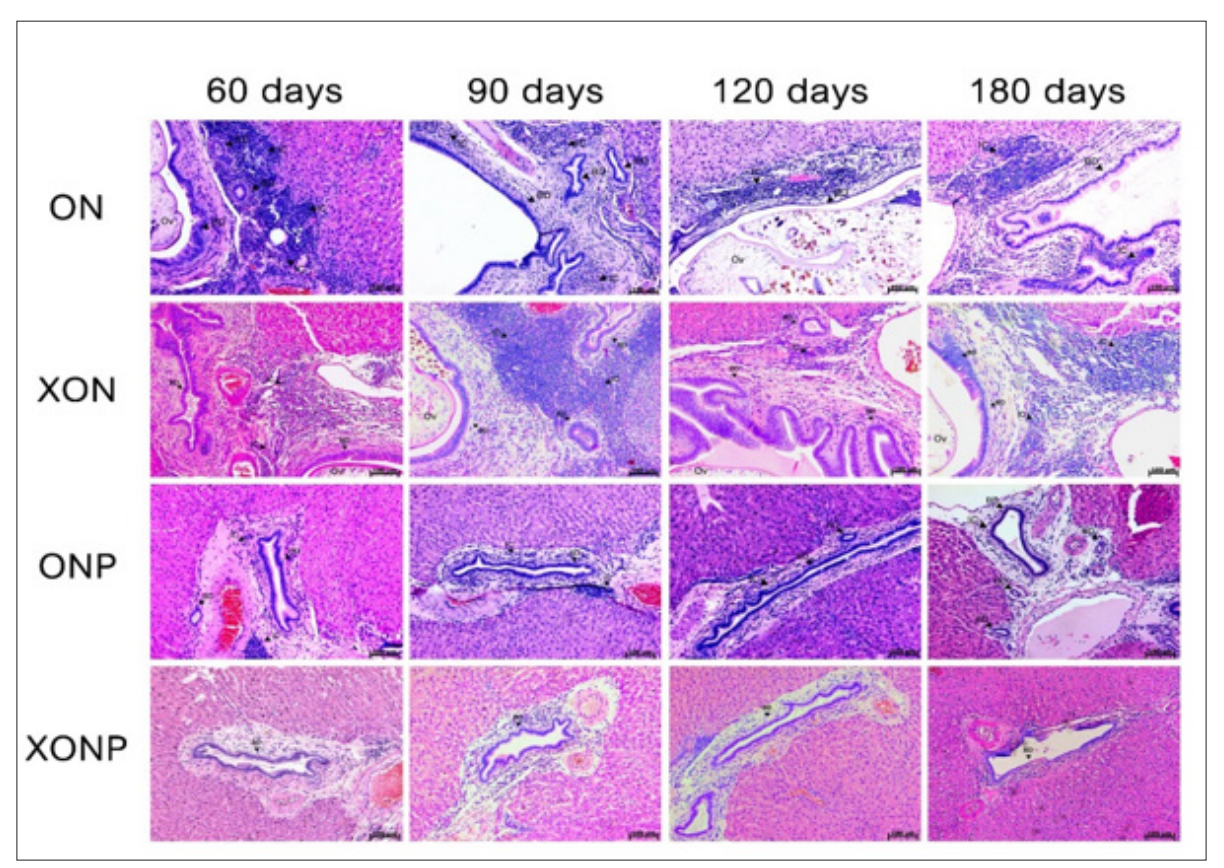

Figure 1: The histopathology changes of the group infected with Ov and administered with NDMA (ON), presence of xanthohumol (XON), presence of praziquantel (ONP) and presence of xanthohumol and praziquantel (XONP) groups at 60, 90, 120, 180 days respectively that examined by hematoxylin and eosin (H\&E) staining in CCA genesis hamsters. An original magnification is $\times 10$ for all figures. $\mathrm{BD}=$ Bile duct hyperplasia, $\mathrm{IC}=$ Inflammatory cells area, and Ov $=$ Opisthorchis viverrini.

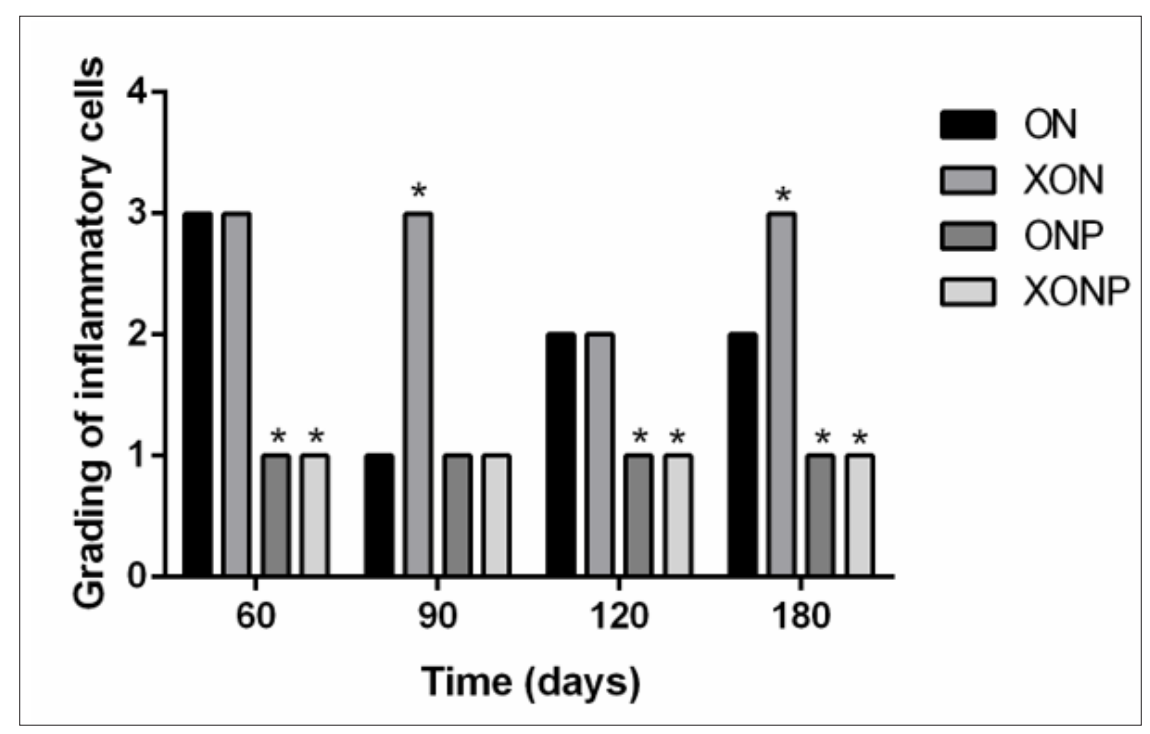

Figure 2: Bar graph comparisons about grading of inflammatory cells in each group of hamster liver; Grade 1: few, Grade 2: moderate, Grade 3: numerous. ${ }^{*} \mathrm{P}<0.05$ compared to the ON group.

The Inhibitory Effects of XN With or Without PZQ Supplements on Twist Expression in Hamster Model

Examples of the immunohistochemical staining results for Twist expression in studied groups as shown in Figure 3. Twist expression was presented in nucleus as well as cytoplasm of hepatocyte, hepatic bile duct and hyperplasia. Our results showed that higher Twist expression was observed along CCA genesis in ON group with time dependent manner. The strongest staining of
Twist was found at day 180 in hyperplasia area of the bile duct epithelia in ON group. Interestingly, the reduction of Twist was seen in XON and ONP groups at day 90 when compared with the ON group. Moreover, our results exhibited an effective reduction of Twist expression around bile duct area for the XONP group when compared to all other groups. This was particularly evident in the expression of Twist in XONP group which was reduced from day 90. 


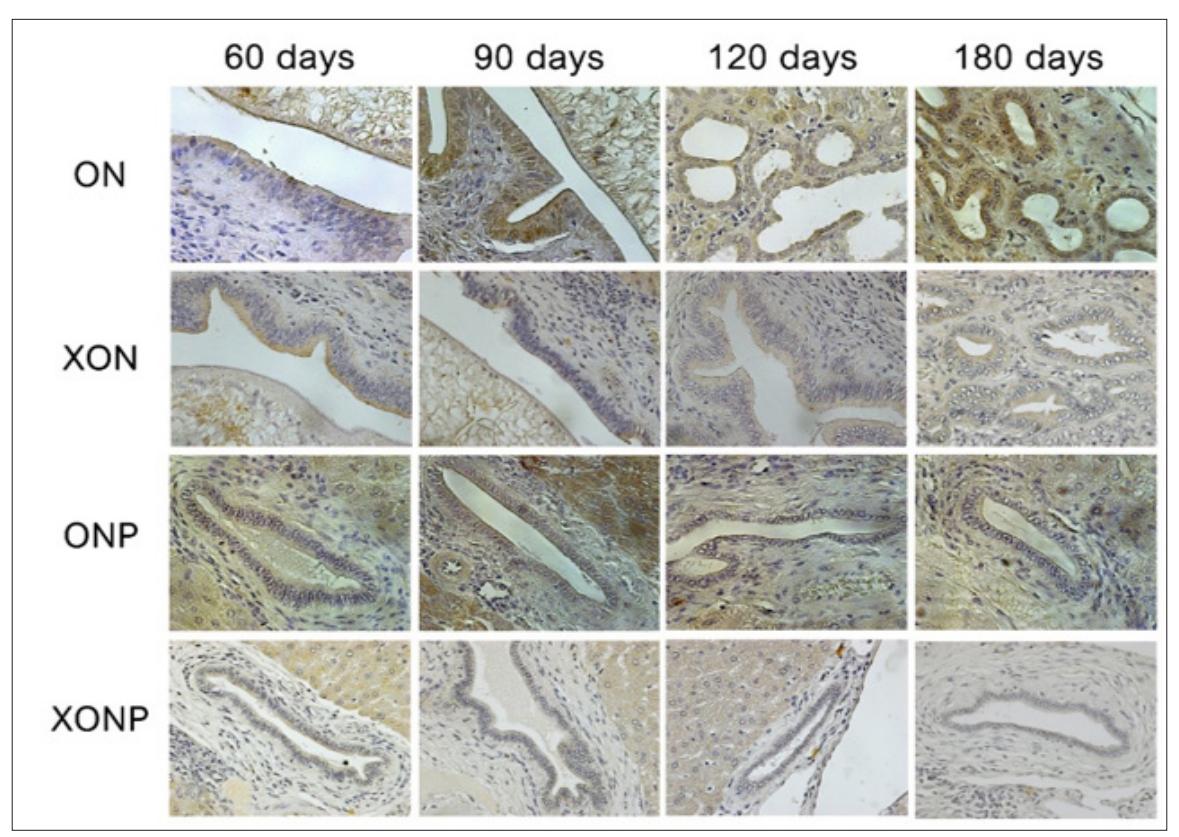

Figure 3: Immunohistochemical staining for Twist expression in the group infected with Ov and administered with NDMA $(\mathrm{ON})$, presence of xanthohumol $(\mathrm{XON})$, presence of praziquantel (ONP) and presence of xanthohumol and praziquantel (XONP) groups at $60,90,120,180$ days respectively. An original magnification is $\times 40$ for all figures.

\section{Discussion}

At present, the medical approaches for diagnosis, prevention and treatment of CCA are incompetent and establishing novel potential therapeutic ways for CCA should be attempted. Characterization and use of effective natural chemopreventive agents that undertaken by preventing various mechanisms for cancer development have become important issues in cancer researches. Many works informed the anti-carcinogenic features of XN, a natural prenylated chalcone from hop, with a phenomenal broad-spectrum that has distinct inhibitory function in carcinogenesis at the initiation, promotion, and progression stages. Persistent with anti-cancer capability of XN, it strongly regulates the enzymes activity related in detoxification and metabolism of carcinogen. Furthermore, this substance is competent to scavenge oxidative stress as reactive oxygen species and to restrict the production of superoxide anion radical and nitric oxide [25]. In CCA, XN has the inhibitory effects of proliferation and apoptosis in human CCA cell lines through the Notch1/Akt signaling pathway [26].

Additionally in vivo and in vitro study, $\mathrm{XN}$ can inhibit the activation of STAT3 in accordance with the Akt-NFKB signaling pathway suppression [26]. Taken together, these data suggested the potential application of $\mathrm{XN}$ as a novel, impressively available chemopreventive agent to become a beneficial novel medical approach and strategies for the CCA prevention and treatment. We then investigated the effect of XN on the alteration of histopathology to assess the inflammation in CCA-induced hamster animal model. Our results showed the extensive aggregation of inflammatory cells was found in the group of $\mathrm{Ov}$ and NDMA treated hamsters (ON).
This related to many works that has been showed the liver fluke can induce chronic inflammatory response, Sripa and coworkers revealed the presence of the $\mathrm{Ov}$ antigens was associated with heavy inflammatory cell infiltration, particularly with mononuclear cells [27]. In the treatment of XN alone (XON) found that XN could not reduce the aggregation of inflammatory cells around the hepatic bile ducts but there was an increasing trend of inflammatory cells on day $60,90,120,180$ respectively when compared with ON group.

The possible explanation about the type of inflammatory cells that we observed in XON group is M1 macrophages which are normally activated by IFN- $\gamma$ or lipopolysaccharide (LPS), and produce pro-inflammatory cytokines, phagocytize microbes, and initiate an immune response [28] more than M2 macrophages, tumor-associated macrophages (TAMs) which are actively promote tumor growth [29]. Moreover, our preliminary results showed that M1 macrophages was increased in XON group (data not shown). However, studies should be conducted in depth to prove this hypothesis. In ONP group which was PZQ treated hamsters, the results showed that PZQ could reduce the number of inflammatory cells around the bile duct as well on day 60 and the observation was clear especially on day 90,120 , and 180 respectively. When used $\mathrm{XN}$ together with the PZQ (XONP) group it was found to be more effective in reducing inflammatory cells in hamster model.

For the reason that PZQ is effective anthelmintic to eradicate Ov resulting in immune response and inflammatory process were not happened including inflammatory cells. Pinlaor and coworkers indicated that Ov elimination by PZQ treatment reduced bile duct inflammation and oxidative and nitrative DNA damage in acute 
stage or early stage of opisthorchiasis [30,31]. At present, EMT has been recognized as a potential mechanism related with various types of cancer development. Many works have shown the role of EMT regulators involve in cancer progression and one of these EMT regulator is Twist, it was identified as one of the most key transcription factors that is up-regulated in metastatic tumors [7]. From our immunohistochemical results showed that Twist is overexpressed in ON group at the time dependent manner from day 60 and strongly expressed on day 180. In contrast, the expression of Twist is decreased and absented in the hamster group that treated with XN, PZQ and combination. These results suggested that XN in combination with PZQ could suppress the EMT process by reduced EMT transcription factor expression, Twist efficiently.

According to the study of XN correlated with EMT showed that XN can suppress protein markers of EMT and cell mobility for instance paxillin, MCL2 and S100A4 [32]. The Twist expression results are similar to inflammatory results in experimented hamster group that have mentioned before. Taken together, there was an evidence that Twist was correlated with inflammation in various ways, Twist1 and Twist2 are key regulators of B cell activation in an inflammatory environment such as autoimmune disease [33]. Correspondingly, IFN-induced Twist1 expression was demonstrated to inhibit TNF- $\alpha$ production in macrophages, supporting in down-regulating the inflammatory response [14]. The results of the current study show that XN alone could not reduce inflammatory lesions around bile duct epithelial cells but in combination with PZQ can effectively decrease of inflammatory cell in liver. For EMT, XN and/or PZQ could potentially reduce Twist expression. Further studies are needed to confirm in depth the association between Twist, an EMT regulator and CCA prevention.

\section{Acknowledgement}

This study was supported by the grant from Khon Kaen University to Anchalee Techasen under grant 591004; we appreciated to Mr. Chakrit Chawarit for laboratory practice on $\mathrm{H} \& \mathrm{E}$ study.

\section{References}

1. Sripa B, Kaewkes S, Sithithaworn P, Mairiang E, Laha T, et al. (2007) Liver fluke induces cholangiocarcinoma. Plos medicine 4(7): 201.

2. Yongvanit P, Pinlaor S, Bartsch H (2012) Oxidative and nitrative DNA damage: key events in opisthorchiasis-induced carcinogenesis. Parasitology international 61(1): 130-135.

3. Treeprasertsuk S, Poovorawan K, Soonthornworasiri N, Chaiteerakij $\mathrm{R}$, Thanapirom K, et al. (2017) A significant cancer burden and high mortality of intrahepatic cholangiocarcinoma in Thailand: a nationwide database study. BMC gastroenterology 17(1): 3.

4. Hughes T, O'Connor T, Techasen A, Namwat N, Loilome $\mathrm{W}$, et al. (2017) Opisthorchiasis and cholangiocarcinoma in Southeast Asia: an unresolved problem. International journal of general medicine 10: 227237.

5. Sripa B, Tangkawattana S, Brindley PJ (2018) Update on Pathogenesis of Opisthorchiasis and Cholangiocarcinoma. Advances in parasitology 102: 97-113.

6. Thiery JP, Acloque H, Huang RY, Nieto MA (2009) Epithelial-mesenchymal transitions in development and disease. Cell 139(5): 871-890.
7. Yang J, Mani SA, Donaher JL, Ramaswamy S, Itzykson RA, et al. (2004) Twist, a master regulator of morphogenesis, plays an essential role in tumor metastasis. Cell 117(7): 927-939.

8. Shibata K, Kajiyama H, Ino K, Terauchi M, Yamamoto E, et al. (2008) Twist expression in patients with cervical cancer is associated with poor disease outcome. Annals of oncology 19(1): 81-85.

9. Kwok WK, Ling MT, Lee TW, Lau TC, Zhou C, et al. (2005) Up-regulation of TWIST in prostate cancer and its implication as a therapeutic target. Cancer research 65(12): 5153-5162.

10. Kyo S, Sakaguchi J, Ohno S, Mizumoto Y, Maida Y, et al. (2006) High Twist expression is involved in infiltrative endometrial cancer and affects patient survival. Human pathology 37(4): 431-438.

11. Niu RF, Zhang L, Xi GM, Wei XY, Yang Y, et al. (2007) Up-regulation of Twist induces angiogenesis and correlates with metastasis in hepatocellular carcinoma. Journal of experimental \& clinical cancer research : CR 26(3): 385-394.

12. Kang Y, Massague J (2004) Epithelial-mesenchymal transitions: twist in development and metastasis. Cell 118(3): 277-279.

13. Duangkumpha K, Techasen A, Loilome W, Namwat $N$, Thanan $R$, et al. (2014) BMP-7 blocks the effects of TGF-beta-induced EMT in cholangiocarcinoma. Tumour biology: The journal of the International Society for Oncodevelopmental Biology and Medicine 35(10): 96679676.

14. Sharif MN, Sosic D, Rothlin CV, Kelly E, Lemke G, et al. (2006) Twist mediates suppression of inflammation by type I IFNs and Axl. The Journal of experimental medicine 203(8): 1891-1901.

15. Chen QH, Fu ML, Chen MM, Liu J, Liu XJ, et al. (2012) Preparative isolation and purification of xanthohumol from hops (Humulus lupulus L.) by high-speed counter-current chromatography. Food chemistry 132(1): 619-623.

16. Dorn C, Weiss TS, Heilmann J, Hellerbrand C (2010) Xanthohumol, a prenylated chalcone derived from hops, inhibits proliferation, migration and interleukin-8 expression of hepatocellular carcinoma cells. International journal of oncology 36(2): 435-441.

17. Drenzek JG, Seiler NL, Jaskula Sztul R, Rausch MM, Rose SL (2011) Xanthohumol decreases Notch1 expression and cell growth by cell cycle arrest and induction of apoptosis in epithelial ovarian cancer cell lines. Gynecologic oncology 122(2): 396-401.

18. Ferk F, Huber WW, Filipic M, Bichler J, Haslinger E, et al. (2010) Xanthohumol, a prenylated flavonoid contained in beer, prevents the induction of preneoplastic lesions and DNA damage in liver and colon induced by the heterocyclic aromatic amine amino-3-methylimidazo[4,5-f] quinoline (IQ). Mutation research 691(1): 17-22.

19. Deeb D, Gao X, Jiang H, Arbab AS, Dulchavsky SA, et al. (2010) Growth inhibitory and apoptosis-inducing effects of xanthohumol, a prenylated chalone present in hops, in human prostate cancer cells. Anticancer research 30(9): 3333-3339.

20. Saito K, Matsuo Y, Imafuji H, Okubo T, Maeda Y, et al. (2018) Xanthohumol inhibits angiogenesis by suppressing nuclear factor-kappaB activation in pancreatic cancer. Cancer science 109(1): 132-140.

21. Dokduang H, Yongvanit P, Namwat N, Pairojkul C, Sangkhamanon S, et al. (2016) Xanthohumol inhibits STAT3 activation pathway leading to growth suppression and apoptosis induction in human cholangiocarcinoma cells. Oncology reports 35(4): 2065-2072.

22. Jamnongkan W, Thanee M, Yongvanit P, Loilome W, Thanan R, et al. (2018) Antifibrotic effect of xanthohumol in combination with praziquantel is associated with altered redox status and reduced iron accumulation during liver fluke-associated cholangiocarcinogenesis. PeerJ 6: e4281.

23. Gerhauser C (2005) Broad spectrum anti-infective potential of xanthohumol from hop (Humulus lupulus L.) in comparison with activities of other hop constituents and xanthohumol metabolites. 
Molecular nutrition \& food research 49(9): 827-831.

24. Goodman ZD (2007) Grading and staging systems for inflammation and fibrosis in chronic liver diseases. Journal of hepatology 47(4): 598-607.

25. Gerhauser C, Alt A, Heiss E, Gamal Eldeen A, Klimo K (2002) Cancer chemopreventive activity of Xanthohumol, a natural product derived from hop. Molecular cancer therapeutics 1(11): 959-969.

26. Walden D, Kunnimalaiyaan S, Sokolowski K, Clark TG, Kunnimalaiyaan $M$ (2017) Antiproliferative and apoptotic effects of xanthohumol in cholangiocarcinoma. Oncotarget 8(50): 88069-88078.

27. Sripa B, Kaewkes S (2000) Localisation of parasite antigens and inflammatory responses in experimental opisthorchiasis. International journal for parasitology 30(6): 735-740.

28. Thanee M, Loilome W, Techasen A, Namwat N, Boonmars T, et al. (2015) Quantitative changes in tumor-associated M2 macrophages characterize cholangiocarcinoma and their association with metastasis. Asian Pacific journal of cancer prevention 16(7): 3043-3050.

29. Lewis CE, Pollard JW (2006) Distinct role of macrophages in different tumor microenvironments. Cancer research 66(2): 605-612.

\section{ISSN: 2574-1241}

DOI: $10.26717 / B J S T R .2019 .12 .002284$

Anchalee Techasen. Biomed J Sci \& Tech Res

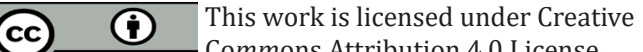

Submission Link: https://biomedres.us/submit-manuscript.php
30. Pinlaor S, Prakobwong S, Hiraku Y, Kaewsamut B, Dechakhamphu S, et al. (2008) Oxidative and nitrative stress in Opisthorchis viverrini-infected hamsters: an indirect effect after praziquantel treatment. The American journal of tropical medicine and hygiene 78(4): 564-573.

31. Pinlaor S, Prakobwong S, Boonmars T, Wongkham C, Pinlaor P, et al. (2009) Effect of praziquantel treatment on the expression of matrix metalloproteinases in relation to tissue resorption during fibrosis in hamsters with acute and chronic Opisthorchis viverrini infection. Acta tropica 111(2): 181-191.

32. Viola K, Kopf S, Rarova L, Jarukamjorn K, Kretschy N, et al. (2013) Xanthohumol attenuates tumour cell-mediated breaching of the lymphendothelial barrier and prevents intravasation and metastasis. Archives of toxicology 87(7): 1301-1312.

33. Doreau A, Belot A, Bastid J, Riche B, Trescol Biemont MC, et al. (2009) Interleukin 17 acts in synergy with B cell-activating factor to influence $B$ cell biology and the pathophysiology of systemic lupus erythematosus. Nature immunology 10(7): 778-785.

$\begin{array}{ll}\text { BIOMEDICAL } & \text { Assets of Publishing with us } \\ \text { RESEARCHES } & \text { - Global archiving of articles } \\ \text { - Immediate, unrestricted online access }\end{array}$

Cite this article: Phongsaran K, Wassana J, Malinee T, Watcharin L, Anchalee T. Anti-Inflammatory Effect of Xanthohumol in Combination With 